\section{BRAZZILIAN JOURNAL \\ OF MEDICAL AND BIOLOGICAL RESFARCH}

www.bjournal.com.br
ISSN 0100-879X

Volume 42 (8) 692-775 August 2009

CLINICAL INVESTIGATION

Braz J Med Biol Res, August 2009, Volume 42(8) 722-730

Circadian cardiac autonomic function in perinatally

HIV-infected preschool children P.R. Benchimol-Barbosa

The Brazilian Journal of Medical and Biological Research is partially financed by
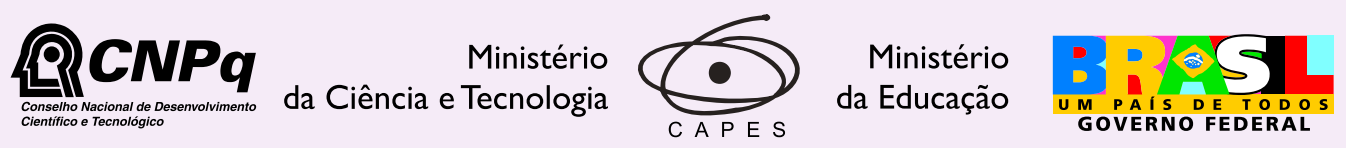

TFAPESP

Institutional Sponsors 


\title{
Circadian cardiac autonomic function in perinatally HIV-infected preschool children
}

P.R. Benchimol-Barbosa

Serviço de Cardiologia, Universidade do Estado do Rio de Janeiro, and Serviço de Cardiologia, Corpo de Bombeiros Militar do Estado do Rio de Janeiro, Rio de Janeiro, RJ, Brasil

\begin{abstract}
The 24-h heart rate variability and QT-interval adaptation was investigated in perinatally HIV-infected preschool children classified according to immunological status in order to assess autonomic function at early stages of infection. Thirty-five perinatally HIV-infected and clinically stable children ( $4.8 \pm 0.3$ years) were enrolled after approval of the study by the University Hospital Pedro Ernesto Ethics Committee and written informed parental consent was obtained. The children were classified according to peripheral CD4+ count (cells/ $\mu \mathrm{L})$ as follows: group 1, $N=11$ ( $\geq 1000)$; group 2, $N=7(\geq 500$ and $<1000)$; group 3, $N=17(<500)$. Left ventricular ejection fraction (>55\%), 24-h RR interval variability (RRV) indexes (NN, SDANN, SDNN index, r-MSSD) and 24-h QT and Bazett-corrected QT (QTc) were determined, and groups were matched for age, body surface area, and left ventricular ejection fraction, reducing biases in RRV. The peak differences $(\Delta)$ between the highest and lowest RRV and QT indexes were extracted from nocturnal (1 am- $6 \mathrm{am}$ ) and daytime (1 pm-6 pm) hourly assessed segments, respectively. Pearson's correlation ( $r$ ) and Kruskal-Wallis ANOVA were used to compare groups. CD4+ count correlated positively with $\Delta N N(r=0.45 ; P=0.003)$. There were no significant differences in daytime NN among groups. Nighttime SDNN index $(P=0.01)$, nighttime $r-M S S D(P$ $=0.003), \Delta N N(P=0.01), \Delta S D N N$ index $(P=0.03)$ and $\Delta r-M S S D(P=0.004)$ were significantly lower in group 3 than in the other groups. Expected nighttime QTc-interval lengthening was not observed in all groups. In perinatally HIV-infected preschool children with preserved left ventricular systolic function, parasympathetic-mediated autonomic dysfunction parallels immune status, impairing both RRV and circadian QTc interval adaptation.
\end{abstract}

Key words: Autonomic nervous system; Circadian rhythm; Heart rate variability; Human immunodeficiency virus infection; Perinatally HIV-infected children

\section{Introduction}

Cardiac structural and functional abnormalities are frequently observed in children infected with human immunodeficiency virus (HIV) (1-3), although their clinical presentation may be irrelevant and symptoms are often attributed to diverse organic systems (4-6). Nevertheless, amongst cardiac abnormalities detected in children with acquired immune deficiency syndrome, mild systolic and diastolic impairments, autonomic nervous system disturbances, cardiac arrhythmias and sudden death have been noteworthy (7-13). On the other hand, some echocardiographic abnormality has been detected in 1 of $4 \mathrm{HIV}$-positive children and data regarding cardiac autonomic function in the pediatric population are lacking (14-16). Furthermore, the interaction between the immune and the autonomic nervous systems has been extensively explored both in vitro and in vivo, indicating the presence of a causal interrelationship between the two systems $(17,18)$. In particular, the development and proliferation of CD4+ cells, a class of immune cells particularly important in host defense against HIV, seems to be influenced by a direct sympathetic effect on $\beta_{2}$ adrenergic surface receptors and further intracellular messengers and, indirectly, by parasympathetic influence

Correspondence: P.R. Benchimol-Barbosa, Serviço de Cardiologia, Boulevard 28 de Setembro, 77, 2ํo andar, 20551-900 Rio de Janeiro, RJ, Brasil. E-mail: ecgar@yahoo.com

Research partially supported by Faculdade de Ciências Médicas, Universidade do Estado do Rio de Janeiro (\#5152/2001), Rio de Janeiro, RJ, Brasil.

Received August 3, 2008. Accepted June 1, 2009. 
on adrenergic organs, potentially having a relevant impact on the HIV population.

The aim of present study was to investigate cardiac autonomic function in perinatally HIV-infected children using 24-h ambulatory ECG RR interval and QT interval variability indexes and to correlate these data with current immune status.

\section{Material and Methods}

In a cross-sectional study, a cohort of 35 perinatally HIVinfected preschool children was enrolled for assessment of cardiac autonomic function. HIV infection was diagnosed by history of maternal HIV serum positivity and confirmation in children by both immune-enzymatic (ELISA) and Western blot assays since birth. Data were collected in a prospective manner and, on admission, children were (mean \pm SEM) $4.7 \pm 0.3$ years old, 16 were males, body surface area was $0.69 \pm 0.02 \mathrm{~m}^{2}$, and all were in regular outpatient clinic follow-up at 3- to 6-month intervals in the Pediatric and Infective Diseases Department of University Hospital Pedro Ernesto (State University of Rio de Janeiro, Brazil) with the same team of physicians since birth. All children received highly active antiretroviral therapy according to clinical stage and CD4+ count, as part of the program of the Brazilian Health Ministry. Compliance with antiretroviral drug therapy, defined as percent children under regular medication treatment, was assessed with parents or legal representatives by means of a simplified questionnaire. Blood sample analyses were carried out in all children for viral load, CD4+ count, CD8+ count and hemoglobin tests at enrollment. The University Hospital Pedro Ernesto Ethics Committee under registration number 503/2000 approved the study protocol and the parents or legal representatives gave written informed consent. The study protocol followed the principles of the Declaration of Helsinki.

On admission, children were staged according to peripheral blood CD4+ count based on the 1994 revision criteria of the classification of the Centers for Disease Control and Prevention (Atlanta, USA) for HIV infection. They were divided into three groups: group 1, 11 children with a CD4+ count $\geq 1000$ cells $/ \mu L(1225 \pm 109)$; group 2,7 children with a CD4+ count $<1000$ and $\geq 500$ cells $/ \mu L(778 \pm 199)$, and group 3, 17 children with a CD4+ count $<500$ cells $/ \mu L$ (391 $\pm 96 ; \mathrm{P}<0.001)$. All groups were matched for age, body surface area (age-corrected Mosteller's monogram formula) (19), and global left ventricular systolic function in order to reduce potential interference with autonomic function assessment. All children were clinically stable.

Exclusion criteria were: clinical and laboratory evidence of active infection, congenital heart disease, intraventricular conduction disturbances, diabetes mellitus, and thyroid or hepatic dysfunction.

\section{1-D/2-D echocardiogram}

Echocardiographic evaluations were carried out with the patient in left lateral decubitus, at $25^{\circ} \mathrm{C}$ in a half-light environment, using ATL APOGEE CX200 (Interspec-ATL, USA) and 2.5 to $2.75 \mathrm{MHz}$ transducers. Echocardiographic images were obtained simultaneously with ECG monitoring according to the recommendations of both the American Society of Echocardiography (20) and the Brazilian Society of Echocardiography (21). Final left ventricular diastolic diameter and final left ventricular systolic diameter were determined, left ventricular ejection fraction was estimated by the method of Teichholz (22), and left ventricular mass by the formula of Devereux (23). An echocardiogram was obtained for each child within one month after admission.

\section{Twenty-four-hour ambulatory electrocardiogram and RR interval variability}

Twenty-four-hour ambulatory ECG was acquired using a two-channel Dynacord model 420 recorder (DMS-Diagnostic Monitoring System, USA) and analyzed with an Altair PC Holter System version 6.00 software (USA) at a $200 \mathrm{~Hz} /$ channel sampling rate. Careful reviews of the 24-h ambulatory ECG records were carried out by a trained observer blind to the children's clinical conditions within 1 month after admission and using software interactive editing facilities in order to exclude arrhythmia and ECG artifacts.

Twenty-four-hour ambulatory ECG records were then separated into 24 non-overlapping 1-h segments for the analysis of RR interval variability (RRV) in the time domain using a protocol described elsewhere (24). Each 1-h segment was previously divided into 5-min non-overlapping epochs, from which consecutive normal RR intervals (mean $\pm \mathrm{SD}$ ) were extracted. The following conventional variables were extracted from 24-h ambulatory ECG: NN (hourly mean of normal RR intervals), SDANN (hourly standard deviation of the mean of normal RR intervals), SDNN index (hourly mean of the standard deviation of normal RR intervals), and r-MSSD (hourly mean of root mean square successive difference of normal RR intervals). A significant correlation was observed between SDNN index and r-MSSD throughout the 24-h period within all groups (group 1: 0.96, $\mathrm{P}<0.001$; group 2: 0.94, $\mathrm{P}<0.001$; group 3: 0.87, $\mathrm{P}<$ $0.001)$. Maximal night-to-day cycle [circadian variation, $(\Delta)$ ] was quantitatively determined over a $24-\mathrm{h}$ period by extracting RRV variables from 1-h segment, respectively, corresponding to the highest and the lowest NN estimates. Hourly SDANN, SDNN index, and r-MSSD estimates were arbitrarily extracted from segments corresponding to the 
respective zenith and nadir points of NN estimates in a 24-h cycle. The rationale for this approach was based on the fact that RRV indexes are strongly dependent on average NN estimates (24), thereby making uniform the period of the day when variables are extracted for analysis. Thus, larger NN estimates were expected to be associated with larger estimates of each RRV variable analyzed. In fact, a positive correlation between RRV indexes and average $R R$ intervals assessed during 1-h periods was observed for all groups (overall average Pearsons' correlation coefficient $0.57 \pm 0.01 ; P<0.001)$.

\section{Ventricular repolarization analysis}

Ventricular repolarization duration was assessed hourly in all children. The QT interval was estimated by the average of the QT interval of three consecutive normal cardiac beats (MQT). Beats for QT interval measurements were selected so that the variation of the preceding $R R$ interval duration was within $5 \%$ of the respective 1 -h segment NN. To assess maximal circadian variation of ventricular repolarization duration, heartbeats were extracted from hourly segments corresponding to the respective maximum and minimum NN in the 24-h ambulatory ECG.

$Q T$ interval measurements. The onset ( $Q$-wave onset) and offset (T-wave offset) of QT intervals on selected beats were detected by visual inspection using digital calipers and the beat-editing facilities of the analyzing software by a trained observer blind to the children's clinical conditions. Both onset and offset marks were assessed at the point where the Cartesian curvature at the junction between the baseline and, respectively, the inscription of the Q-wave and the end of the T-wave were locally maximal. The Cartesian curvature describes how sharply (or smoothly) a function changes direction at a particular point. In a continuous and low-noise ECG tracing, starting from a flat baseline point (i.e., P-R or T-P segments) and progressing toward the waveform undulation (i.e., QRS complex or T-wave), the Cartesian curvature progressively increases to the point where the curve maximally bends (maximal curvature point) onto itself and then smoothes out, thus indicating the mark with the sharpest contour. The baseline either before the onset mark or after the offset mark was stable for at least $2 \mathrm{~ms}$. QT intervals were heart rate corrected according to Bazett's formula (25) to assess average corrected QT interval (MQTc).

\section{Statistical analyses}

Numerical and categorical variables are reported as means \pm SEM or as rates or percent, as appropriate. Numerical variable analyses were carried out for the interimmunological groups by Kruskal-Wallis ANOVA and the least squared difference contrast test. Categorical variable analyses were carried out by the Yates-corrected chi-square test or the Fisher exact test, when appropriate. The Pearson coefficient was used to assess the correlation between variables, and the Student $t$-test was applied to correlation analysis.

In all 24-h ambulatory ECG records, hourly NN were correlated with SDANN, SDNN index, and r-MSSD within immunological groups. SDNN index and r-MSSD were assessed as parasympathetic modulation indexes, whereas SDANN was assessed as a sympathetic autonomic modulation index.

The level of significance was set at 0.05. Statistical analyses were carried out using MS-Excel 2000 (Microsoft Corporation, USA), Stratigraphics Plus version 5.1 (Statistical Graphics Corporation, USA) and Epi-Info 6.04b (Centers for Disease Control and Prevention, USA).

\section{Results}

Adhesion to antiretroviral drug therapy was higher in groups 1 (82\%) and $2(100 \%)$ compared to group 3 (41\%; $P=0.01)$.

Age (group 1: $4.2 \pm 0.5$ years; group 2: $4.8 \pm 0.7$ years; group 3: $5.0 \pm 0.4$ years), gender (female/male: group 1: 7/4; group 2: 5/2; group 3: 7/10), body surface area (group 1: $0.72 \pm 0.03$; group 2: $0.75 \pm 0.05$; group 3: $0.68 \pm 0.04$ $\mathrm{m}^{2} ; \mathrm{P}=0.31$ ), and left ventricular function (group 1: $68 \pm$ 2; group 2: $71 \pm 3$; group 3: $66 \pm 2 \%$ ) parameters did not differ significantly among groups. Peripheral CD8+ count was also not significantly different among groups (group 1: $1645 \pm 184$; group 2: $1308 \pm 158$; group 3: $1770 \pm 525$ cells $/ \mu \mathrm{L}$ ). Viral load (group 1: $108 \pm 50$; group 2: $33 \pm 33$; group 3: $244 \pm 101 \times 10^{3}$ copies/ $\mu \mathrm{L}$ ) and peripheral blood hemoglobin concentration (group 1: $10.8 \pm 0.3$; group 2: $11.2 \pm$; group 3: $10.2 \pm 0.2 \mathrm{~g} / \mathrm{dL}$ ) did not differ significantly among study groups.

Furthermore, left ventricular mass (group 1: $37 \pm 3$; group 2: $39 \pm 5$; group 3: $44 \pm 3 \mathrm{~g}$ ), left ventricular end diastolic diameter (group 1: $3.5 \pm 0.1$; group 2: $3.5 \pm 0.1$; group 3: $3.7 \pm 0.1 \mathrm{~cm})$ and left ventricular end systolic diameter (group 1: $2.2 \pm 0.1$; group 2: $2.1 \pm 0.1$; group 3: $2.4 \pm 0.1$ $\mathrm{cm})$ showed similar distributions among groups.

All 24-h ambulatory ECG tapes were successfully analyzed and the overall 24-h artifact incidence corresponded to $1.9 \pm 0.8 \%$ of the period analyzed. The average overall incidence of both supraventricular and ventricular ectopic beats was $0.7 \pm 0.4 / \mathrm{h}$ from 8 am to $8 \mathrm{pm}$ and $0.4 \pm 0.4 / \mathrm{h}$ from $8 \mathrm{pm}$ to $8 \mathrm{am}$.

RRV parameters showed significant circadian variation in all groups. As expected, the RR interval decreased dur- 
ing the night in a similar fashion in all groups. However, some time domain variability indexes showed remarkably different patterns (Figure 1). Hourly averaged NN, SDANN and r-MSSD, and SDNN index graphs for groups 1, 2, and 3 are presented in Figure 1 and Table 1.

After adjustment for daytime NN, the modulation of the parasympathetic system as reported by the SDNN index and r-MSSD was evident in group 1 and remarkably attenuated in group 3 (Table 1). No significant differences in SDANN were detected among the groups.

Peripheral blood CD4+ count correlated positively and viral load correlated negatively with $\Delta N N(r=0.45$ and $r=-0.35$, respectively; Figure 2). CD8+ count showed no significant correlation with night-to-day NN variation $(r=$ -0.13). CD4+, CD8+ and viral load were poorly correlated to $\triangle \mathrm{MQT}(r=0.17, r=-0.18, r=-0.14$, respectively) and $\triangle \mathrm{MQTc}(r=-0.19, r=-0.14, r$ $=0.11$, respectively).

For adjusted daytime average NN in 1-h segment (MNN), circadian $\triangle \mathrm{MNN}, \triangle \mathrm{SDNN}$ index, $\triangle \mathrm{r}$-MSSD and $\triangle \mathrm{MQT}$ interval showed statistically significant differences across groups (Table 1). However, SDANN did not show significant differences across groups, when analyzed in daytime
Table 1. Twenty-four-hour RR-interval variability, and 24-h QT interval parameters of the pediatric patients studied.

\begin{tabular}{lcccl}
\hline RR interval variability and & $\begin{array}{c}\text { Group 1 } \\
\text { QT interval variables }\end{array}$ & $\begin{array}{c}\text { Group 2 } \\
(\mathrm{N}=11)\end{array}$ & $\begin{array}{c}\text { Group 3 } \\
(\mathrm{N}=17)\end{array}$ & $\mathrm{P}^{*}$ \\
\hline Daytime MNN (ms) & $535.8 \pm 25.9$ & $528.7 \pm 22.8$ & $555.3 \pm 10.8$ & \\
Daytime SDNN index (ms) & $51.0 \pm 6.6$ & $42.4 \pm 7.7$ & $41.7 \pm 3.7$ & \\
Daytime r-MSSD (ms) & $39.5 \pm 2.2^{1}$ & $29.2 \pm 1.7^{2}$ & $30.7 \pm 1.6^{1,2}$ & 0.009 \\
Daytime MQT (ms) & $316.2 \pm 12.2$ & $316.9 \pm 4.9$ & $320.7 \pm 5.6$ & \\
Daytime MQTc (ms) & $433.0 \pm 14.3$ & $435.8 \pm 5.9$ & $430.9 \pm 4.4$ & \\
Nighttime MNN (ms) & $762.1 \pm 33.8$ & $711.7 \pm 35.6$ & $692.3 \pm 20.0$ & \\
Nighttime SDNN index (ms) & $94.0 \pm 11.0^{1}$ & $90.7 \pm 18.7$ & $61.2 \pm 6.4^{1}$ & 0.03 \\
Nighttime r-MSSD (ms) & $93.0 \pm 4.1^{1}$ & $73.7 \pm 3.2^{2}$ & $63.7 \pm 5.9^{1,2}$ & 0.003 \\
Nighttime MQT (ms) & $374.9 \pm 12.6$ & $365.7 \pm 8.0$ & $352.7 \pm 8.3$ & \\
Nighttime MQTc (ms) & $429.7 \pm 7.3$ & $435.3 \pm 10.9$ & $424.2 \pm 6.2$ & \\
$\Delta$ MNN (ms) & $226.3 \pm 24.3^{1}$ & $183.0 \pm 24.9$ & $137.1 \pm 26.3^{1}$ & 0.01 \\
$\Delta$ SDNN index (ms) & $43.0 \pm 8.4^{1}$ & $48.3 \pm 15.5^{2}$ & $19.5 \pm 4.1^{1,2}$ & 0.03 \\
$\Delta r-M S S D(m s)$ & $19.5 \pm 4.6^{1}$ & $11.9 \pm 3.8^{2}$ & $-2.0 \pm 1.2^{1,2}$ & 0.004 \\
$\Delta$ MQT (ms) & $58.7 \pm 6.5^{1}$ & $48.8 \pm 11.9$ & $32.0 \pm 7.5^{1}$ & 0.04 \\
$\Delta$ MQTc (ms) & $-3.2 \pm 10.7$ & $-0.5 \pm 14.7$ & $-6.8 \pm 7.4$ & \\
\hline
\end{tabular}

Data are reported as means \pm SEM. $\Delta=$ maximal circadian variation; $N N=$ hourly mean of normal RR intervals; MNN = average NN in 1-h segment; SDNN index = hourly mean of the standard deviation of normal RR intervals; $\mathrm{r}-\mathrm{MSSD}=$ hourly mean of root mean square successive difference of normal RR intervals; MQT (MQTc) = average QT (corrected QT) interval in 1-h segment. *Kruskal-Wallis one-way ANOVA test except for gender comparison where chi-square test with Yates correction was employed. $P$ values greater than 0.05 are not reported. ${ }^{1,2}$ Significant differences $(P<0.05)$ among groups assessed by the least squared difference contrast test.
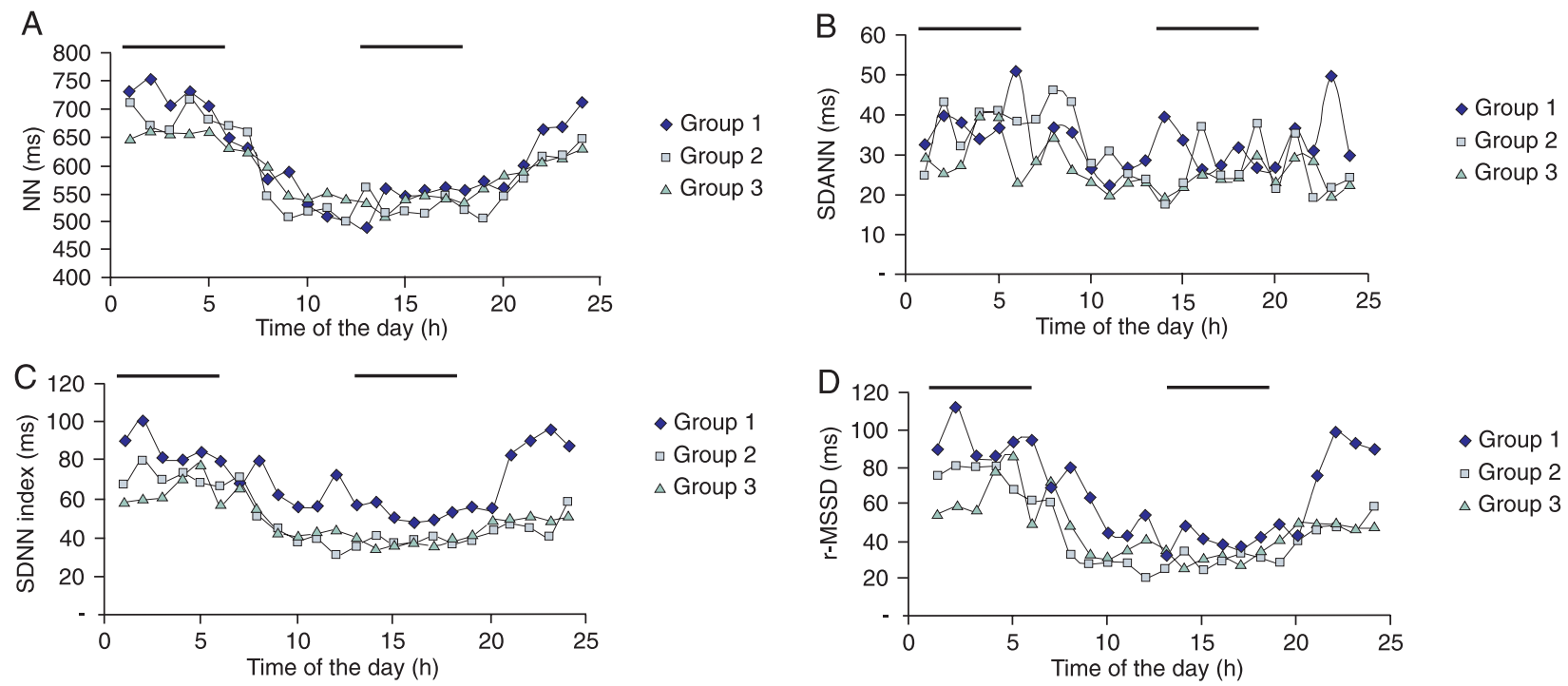

Figure 1. Circadian variation of hourly NN (A), SDANN (B), SDNN index (C), and r-MSSD (D) graphs of group 1 (CD4+ count $\geq 1000$ cells/ $\mu \mathrm{L}$; diamonds), group 2 (CD4+ count $<1000$ and $\geq 500$ cells $/ \mu \mathrm{L} ;$ squares), and group 3 (CD4+ count $<500$ cells $/ \mu \mathrm{L}$; triangles). The horizontal bars on top of the graphs indicate nighttime (1 to $6 \mathrm{am})$ and daytime $(1$ to $6 \mathrm{pm})$ periods. SDANN $=$ hourly standard deviation of the mean of normal RR intervals. See Table 1 for all other abbreviations. 
(group 1: $31.3 \pm 7.3$; group 2: $31.7 \pm 9.7$; group 3: $25.1 \pm 3.7$ $\mathrm{ms}$ ), in nighttime (group 1: $38.1 \pm 4.4$; group 2: $33.1 \pm 4.9$; group 3: $29.7 \pm 3.5 \mathrm{~m}$ ), or in night-to-daytime pattern (group 1: $6.7 \pm 7.4$; group 2: $1.4 \pm 9.7$; group $3: 4.6 \pm 3.8 \mathrm{~ms}$ ). MNN and MQT were positively and significantly correlated $(r=$ 0.82 ; Figure $3 \mathrm{~A})$, as also were $\triangle \mathrm{MNN}$ and $\triangle \mathrm{MQT}(\mathrm{r}=0.53$; Figure 3B), and Bazett-corrected QT interval suppressed the QT to RR interval correlation $(r=-0.01$ and $r=0.18$,
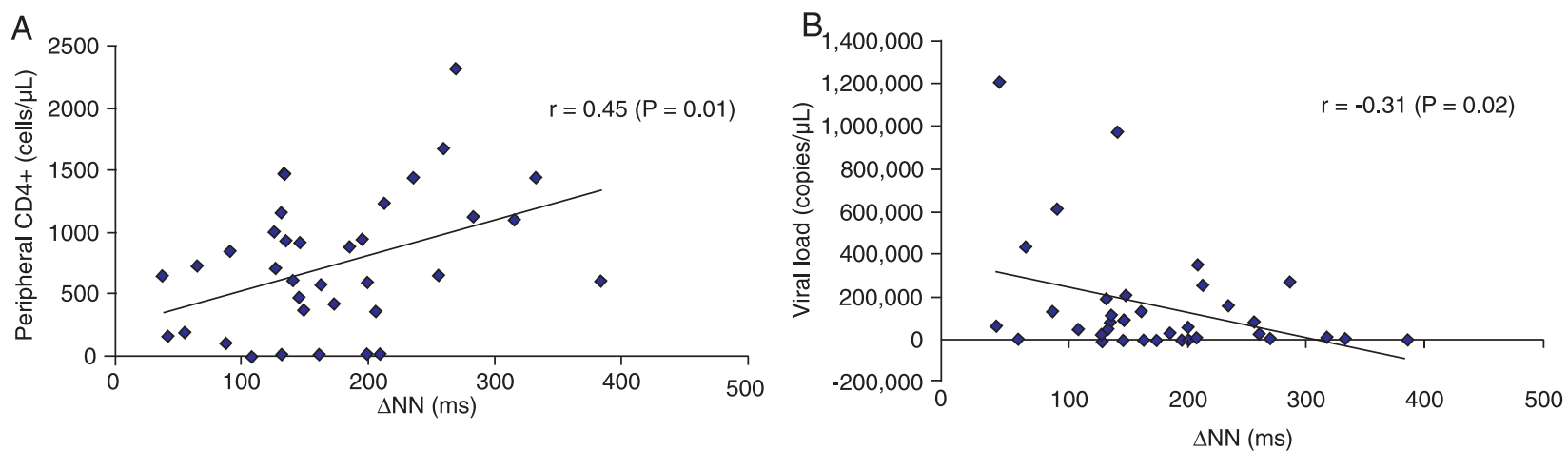

Figure 2. $A$, Plot of CD4+ cell count in a peripheral blood sample vs maximal circadian hourly averaged RR interval variation ( $\Delta \mathrm{NN})$. Note the significant Pearson positive correlation indicating the association between current autonomic status and 24-h cardiac autonomic modulation. $B$, Plot of viral load in a peripheral blood sample vs maximal circadian hourly averaged RR interval variation ( $\Delta \mathrm{NN})$. Note the significant Pearson negative correlation, indicating the association between amount of circulating virus and 24-h cardiac autonomic modulation.

A

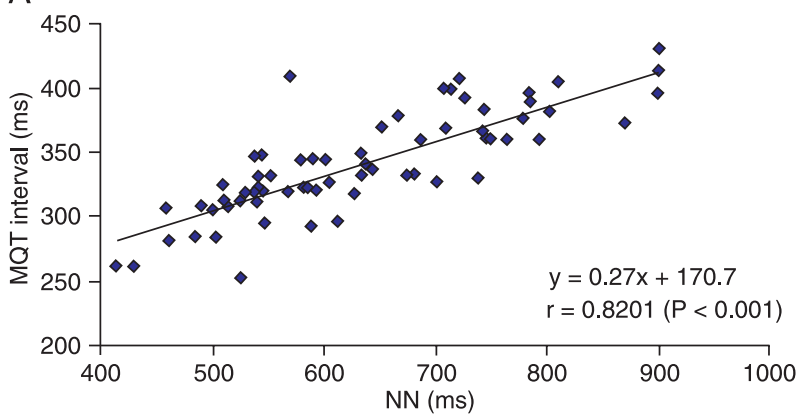

C

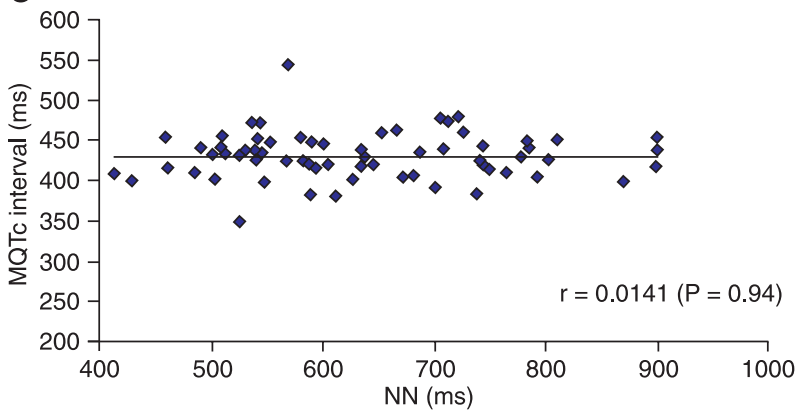

B

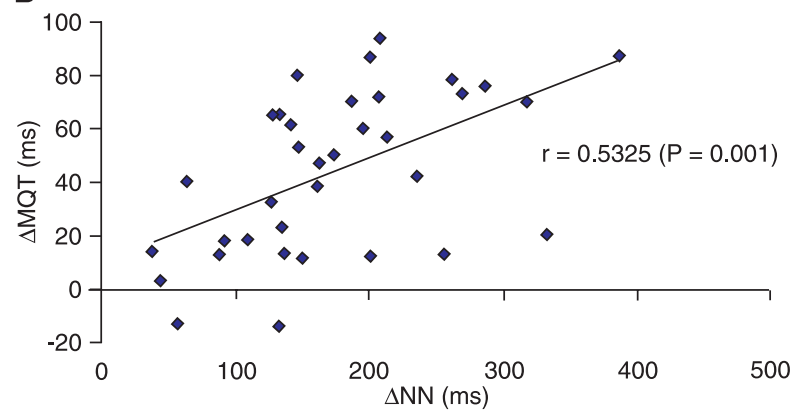

D

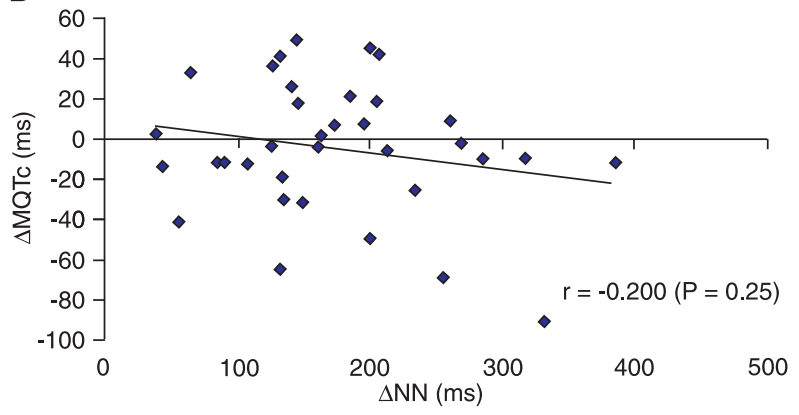

Figure 3. A, Hourly averaged QT intervals (MQT) vs averaged RR intervals (NN). B, Maximal circadian hourly averaged QT interval variation $(\triangle \mathrm{MQT})$ vs maximal circadian $\mathrm{RR}$ interval variation $(\triangle \mathrm{NN})$. Note the significant Pearson positive correlation. $C$, Hourly averaged corrected-QT intervals (MQTc) vs averaged RR intervals (NN). D, Maximal circadian hourly averaged corrected QT interval variation $(\triangle \mathrm{MQTC})$ vs maximal circadian $\mathrm{RR}$ interval variation $(\triangle \mathrm{NN})$. Note in the graphs that, after correction, both $\mathrm{MQTc}$ and $\triangle \mathrm{MQTC}$ became independent of $N N$ and $\triangle N N$, respectively. 
respectively; Figure $3 \mathrm{C}$ and $\mathrm{D})$. No differences regarding daytime or nighttime MQTc interval were observed across groups, and $\triangle \mathrm{MQTC}$ was not significantly different within groups for daytime, nighttime or circadian periods.

\section{Discussion}

In the present study, clinical, echocardiographic, 24-h RR interval variability and 24-h QT interval indexes were analyzed in perinatally HIV-infected children with preserved left ventricular systolic function in a cross-sectional study aiming at assessing cardiac autonomic function. The most relevant findings were: 1) maximal 24-h NN variation directly correlated to peripheral CD4+ count, and 2) cardiac autonomic dysfunction was related to the severity of immune system depression, and was expressed as both predominant parasympathetic dysfunction and repolarization adaptation impairment.

Limited information is available in the literature regarding 24-h cardiac autonomic function in HIV-positive children. Previous studies $(8,14-16)$ have shown a higher prevalence of both heart failure and structural abnormalities related to cardiac autonomic dysfunction in children in advanced stages of HIV infection. Nonetheless, HIV-infected subjects have demonstrated a more pronounced autonomic dysfunction not associated with clinical evidence of heart disease $(26,27)$. Evaluation of the autonomic status of children using the Valsalva maneuver has been carried out, demonstrating a significant autonomic dysfunction, mainly parasympathetic, with either mild or undetectable functional or structural cardiac involvement $(8,14)$. In the present study, groups were matched for age, gender, body surface area and global left ventricular systolic function in order to reduce potential biases in RRV parameters. To the best of our knowledge, this is the first investigation to assess 24-h autonomic function in ambulatory perinatally HIV-infected preschool children.

Although initially unintended, but relevant to the understanding of the current findings, an important social observation from the present study was that compliance with drug therapy schemes was significantly altered in children with more pronounced immune status depression compared with children with more preserved immune status. Although in-depth reasoning is out of the scope of the present study, the psychological distress related to the disease-associated social burden seems to be one of the soundest explanations. In fact, social workers and psychological therapists have provided important support to parents and legally responsible persons regarding their special children's care task.

The autonomic nervous system physiologically adjusts normal inter-beat variation (or RRV) as a consequence of competitive interaction of the parasympathetic and sympathetic stimulation on the sinus node, known as cardiac autonomic modulation $(24,28,29)$. Thus, quantification of RRV can be regarded as a measurement of the autonomicmediated cardiovascular homeostasis, a marker of cardiovascular prognosis $(24,26,27)$. Accordingly, reduction in RRV-related indexes represents autonomic impairment and involves a poor prognosis $(9,24,26,27)$.

In normal children and teenagers, RRV increases with age, reflecting maturation of the autonomic nervous system $(14,15)$. On the other hand, in healthy adults, RRV is negatively correlated with age, reflecting a lifetime progressive loss of autonomic function, known as one of the aspects of aging $(16,17,30,31)$. In the present study, circadian RRV variation was significantly affected by current immunological status, indicating a strong interaction between the autonomic and immune systems. Remarkably, average daytime $\mathrm{NN}$ was fairly constant across groups and $\Delta \mathrm{NN}$ was significantly reduced in group 3 when compared to other groups. Thus, $\Delta \mathrm{NN}$, the maximal circadian (night-to-day) NN variation, was significantly reduced in children presenting a more depressed immune status after adjusting daytime $\mathrm{NN}$. As a part of the strategy employed for analysis, the identification of the maximal night-to-day NN variation in 1-h segments during nighttime and daytime further guided the selection of RRV parameters. The application of the current approach was based on the assumption that average NN strongly affects all RRV parameters (24). In order to reduce a potential bias in determining maximal night-to-day RRV, RRV indexes were arbitrarily measured at the zenith and the nadir of average NN in 1-h segments, thus, reflecting the maximal individual circadian variation.

In a recent study, Nunes (32) described the 24-h RRV behavior of healthy preschool children in Rio de Janeiro, Brazil, using an approach identical to that used in the current study. The author showed a progressive increase in 24-h variation of $\mathrm{NN}$ and $\mathrm{RRV}$ indexes with increasing age, thus confirming the concept of continuous autonomic maturation during the early years of life in the population under study. Comparison of the RRV data obtained in the present study with those of 24 age- and gender-matched control healthy preschool children from Nunes' study (32) revealed that r-MSSD was significantly decreased in group 3. In fact, daytime r-MSSD (Nunes': $45.6 \pm 7.6$ ms vs present study group 3: $30.7 \pm 1.6 \mathrm{~ms}$ ), nighttime r-MSSD (Nunes': $80.9 \pm 12.2$ $\mathrm{ms} v s$ present study group 3: $63.7 \pm 5.9 \mathrm{~ms}$ ), and night-today r-MSSD (Nunes': $35.3 \pm 7.8 \mathrm{~ms}$ vs present study group 3: $-2.0 \pm 1.2 \mathrm{~ms}$ ) were reduced. No significant differences were observed regarding circadian SDANN and SDNN index variation when compared to Nunes' study. These findings 
indicate that in perinatally HIV-infected children, depressed immunity at early ages was related to parasympathetic autonomic system dysfunction. Furthermore, the finding that peripheral CD4+ count positively correlated and viral load negatively correlated with $\triangle N N$ indicates that current immune status and parasympathetic-mediated circadian autonomic modulation may be interdependent. However, it is not clear whether this association is the direct effect of immune dysfunction on the autonomic system or a more general systemic reaction to a state of decreased immunity. In a recent thorough review of the interaction between immunity and the autonomic systems, Nance and Sanders (17) suggested that the parasympathetic system modulates an anti-inflammatory response via a complex interaction with the adrenal medulla and the sympathetic nervous system $(17,18)$. Furthermore, the authors stressed that CD4+ development was affected by activation of the sympathetic nervous system, thus contributing to the reduction of beta receptor density on the cell surface. Although beyond the scope of the present study, it is possible to suggest that decreased parasympathetic activation may have exerted an indirect negative effect on immunity in the present population. However, at present it is not possible to establish whether this effect was transient, and therefore could be reversed, or whether it was the cause or the consequence of a dwelling infectious state. Further studies are warranted to investigate both possibilities.

QT interval adaptation is a measure of cardiac fiber recovery under a continuously changing autonomic demand, and is related to the underlying myocardial functional status. In damaged myocardium, the QT interval does not adapt appropriately to RR interval variation (33) and carries a prospective risk for an adverse outcome. In the present study, both MQT and $\triangle \mathrm{MQT}$ intervals correlated significantly to the $N N$ and $\Delta N N$ intervals, respectively. These findings indicate that repolarization adaptation to heart rate was preserved. Saidi et al. (34) studied the Bazett-corrected QT interval on resting surface ECG in perinatally HIV-infected children from birth to 10 years of age, and observed that average QTc interval showed a significant variation throughout the first decade of life. In the present study, all MQTc intervals were consistently comparable to those reported by Saidi et al. (34) for an equivalent age group. On the other hand, a physiological nighttime prolongation in QTc interval has been observed in healthy adults (35) and healthy children (36), a consequence of increased parasympathetic drive during this period. Adirect parasympathetic-dependent modulation of ventricular repolarization has already been demonstrated both in vitro and in vivo $(37,38)$. In the present study, although MQT was a function of NN in a circadian fashion, no significant nighttime MQTc lengthening was observed in any group, and no significant changes in night-to-day $\triangle M Q T c$ were observed across immune status-classified groups. The finding that HIV infection was associated with both 24-h parasympathetic-mediated RRV and nighttime impairment of QTc interval adaptation supported the view of early HIV infection-dependent parasympathetic dysfunction in this population.

Montague et al. (39) reported a reduced 24-h QTc adaptation in sudden infant death syndrome, which the authors related to increased sympathetic drive. In the present study, no 24-h sympathetic enhancement was observed in any group. Although it has been shown that antiretroviral drugs do not affect the QT or QTc intervals (40), as also found in the present findings, further studies monitoring QT interval adaptation are warranted as new drugs appear on this scenario.

In perinatally HIV-infected preschool children with preserved left ventricular systolic function, cardiac autonomic function parallels immunological status, and is characterized by both attenuation of parasympathetic-mediated circadian RR interval variability and impairment of nighttime QTC interval lengthening.

\section{Limitations of the study}

The analysis of the effect of compliance with specific anti-retrovirus drug schemes on 24-h RRV was not the aim of present study. Although recovery of immune status may parallel RRV recovery in HIV-infected children, the effect of specific drug regimens on heart rate variability still needs assessment.

Additionally, although antiretroviral drugs have been reported not to affect QT or QTc intervals, it was not the aim of the present study to assess the effect of antiviral therapy on circadian QTc interval adaptation. Further studies are needed to investigate the effect of antiviral drugs on circadian QT and QTc interval adaptation in perinatally HIV-infected children.

A potential limitation of the present study was the lack of an age-matched healthy control group. The comparison of RRV indexes between HIV-infected children and a "normal" age-matched control group was based on a large historical cohort study previously carried out by Dr. Nunes (32) in a similar base population from greater Rio de Janeiro city.

\section{Acknowledgments}

I thank Dr. Alfredo S. Bomfim and Eduardo C. Barbosa for their suggestions, Dr. Carolina P. Caldeira and Dr. Ana Teresa Antunes for helping with data analysis, and Dr. Mônica S. Borges for collection of Holter data (Universidade do Estado do Rio de Janeiro). 


\section{References}

1. Grody WW, Cheng L, Lewis W. Infection of the heart by the human immunodeficiency virus. Am J Cardiol 1990; 66: 203206.

2. Rodriguez ER, Nasim S, Hsia J, Sandin RL, Ferreira A, Hilliard BA, et al. Cardiac myocytes and dendritic cells harbor human immunodeficiency virus in infected patients with and without cardiac dysfunction: detection by multiplex, nested, polymerase chain reaction in individually microdissected cells from right ventricular endomyocardial biopsy tissue. Am J Cardiol 1991; 68: 1511-1520.

3. Okoshi MP, Montenegro MR. [Pathology of the heart in AIDS. A study of 73 consecutive necropsies]. Arq Bras Cardiol 1996; 66: 129-133.

4. Herskowitz A, Wu TC, Willoughby SB, Vlahov D, Ansari AA, Beschorner WE, et al. Myocarditis and cardiotropic viral infection associated with severe left ventricular dysfunction in late-stage infection with human immunodeficiency virus. J Am Coll Cardiol 1994; 24: 1025-1032.

5. Lipshultz SE. Dilated cardiomyopathy in HIV-infected patients. N Engl J Med 1998; 339: 1153-1155.

6. De Castro S, Migliau G, Silvestri A, D’Amati G, Giannantoni $P$, Cartoni $D$, et al. Heart involvement in AIDS: a prospective study during various stages of the disease. Eur Heart J 1992; 13: $1452-1459$.

7. Himelman RB, Chung WS, Chernoff DN, Schiller NB, Hollander $\mathrm{H}$. Cardiac manifestations of human immunodeficiency virus infection: a two-dimensional echocardiographic study. J Am Coll Cardiol 1989; 13: 1030-1036.

8. Herskowitz A, Vlahov D, Willoughby S, Chaisson RE, Schulman SP, Neumann DA, et al. Prevalence and incidence of left ventricular dysfunction in patients with human immunodeficiency virus infection. Am J Cardiol 1993; 71: 955-958.

9. Currie PF, Jacob AJ, Foreman AR, Elton RA, Brettle RP, Boon NA. Heart muscle disease related to HIV infection: prognostic implications. BMJ 1994; 309: 1605-1607.

10. Al-Attar I, Orav EJ, Exil V, Vlach SA, Lipshultz SE. Predictors of cardiac morbidity and related mortality in children with acquired immunodeficiency syndrome. J Am Coll Cardiol 2003; 41: 1598-1605

11. Bowles NE, Kearney $\mathrm{DL}, \mathrm{Ni} J$, Perez-Atayde AR, Kline MW, Bricker JT, et al. The detection of viral genomes by polymerase chain reaction in the myocardium of pediatric patients with advanced HIV disease. J Am Coll Cardiol 1999; 34: 857-865.

12. Nogueira G, Macedo AJ, Paixao A, Nunes MA, Ferreira M, Bernardino $\mathrm{L}$, et al. [Cardiovascular morbidity in children with human immunodeficiency virus infection]. Acta Med Port 1998; 11: 1051-1057.

13. Grenier MA, Karr SS, Rakusan TA, Martin GR. Cardiac disease in children with HIV: relationship of cardiac disease to HIV symptomatology. Pediatric AIDS HIV Infect 1994; 5: 174-179.

14. Plein D, Van Camp G, Cosyns B, Alimenti A, Levy J, Vandenbossche JL. Cardiac and autonomic evaluation in a pediatric population with human immunodeficiency virus. Clin Cardiol 1999; 22: 33-36.

15. Freeman R, Roberts MS, Friedman LS, Broadbridge C. Autonomic function and human immunodeficiency virus infection. Neurology 1990; 40: 575-580.

16. Ruttimann S, Hilti P, Spinas GA, Dubach UC. High frequency of human immunodeficiency virus-associated autonomic neuropathy and more severe involvement in advanced stages of human immunodeficiency virus disease. Arch Intern Med 1991; 151: 2441-2443.

17. Nance DM, Sanders VM. Autonomic innervation and regulation of the immune system (1987-2007). Brain Behav Immun 2007; 21: 736-745.

18. Bellinger DL, Millar BA, Perez S, Carter J, Wood C, Thyagarajan S, et al. Sympathetic modulation of immunity: relevance to disease. Cell Immunol 2008; 252: 27-56.

19. Mosteller RD. Simplified calculation of body-surface area. $N$ Engl J Med 1987; 317: 1098.

20. Campos FO, Zielinsky P, Ortiz J, Maciel BC, Andrade JL, Mathias W Jr, et al. [Guideline for indication and utilization of echocardiography in clinical practice]. Arq Bras Cardiol 2004; 82 (Suppl 2): 11-34.

21. Cheitlin MD, Alpert JS, Armstrong WF, Aurigemma GP, Beller GA, Bierman FZ, et al. ACC/AHA guidelines for the clinical application of echocardiography: executive summary. A report of the American College of Cardiology/American Heart Association Task Force on practice guidelines (Committee on Clinical Application of Echocardiography). Developed in collaboration with the American Society of Echocardiography. J Am Coll Cardiol 1997; 29: 862-879.

22. Teichholz LE, Kreulen T, Herman MV, Gorlin R. Problems in echocardiographic volume determinations: echocardiographic-angiographic correlations in the presence of absence of asynergy. Am J Cardiol 1976; 37: 7-11.

23. Salerno M, Esposito V, Farina V, Radetti G, Umbaldo A, Capalbo D, et al. Improvement of cardiac performance and cardiovascular risk factors in children with $\mathrm{GH}$ deficiency after two years of $\mathrm{GH}$ replacement therapy: an observational, open, prospective, case-control study. J Clin Endocrinol Metab 2006; 91: 1288-1295.

24. Heart rate variability. Standards of measurement, physiological interpretation, and clinical use. Task Force of the European Society of Cardiology and the North American Society of Pacing and Electrophysiology. Eur Heart J 1996; 17: 354-381.

25. Indik JH, Pearson EC, Fried K, Woosley RL. Bazett and Fridericia QT correction formulas interfere with measurement of drug-induced changes in QT interval. Heart Rhythm 2006; 3: 1003-1007.

26. Neild PJ, Amadi A, Ponikowski P, Coats AJ, Gazzard BG. Cardiac autonomic dysfunction in AIDS is not secondary to heart failure. Int J Cardiol 2000; 74: 133-137.

27. Mittal CM, Wig N, Mishra S, Deepak KK. Heart rate variability in human immunodeficiency virus-positive individuals. Int $J$ Cardiol 2004; 94: 1-6.

28. Barbosa Filho J, Barbosa PR, Cordovil I. Autonomic modulation of the heart in systemic arterial hypertension. Arq Bras Cardiol 2002; 78: 181-195.

29. Bittencourt MI, Benchimol Barbosa PR, Drumond Neto C, Bedirian R, Barbosa EC, Brasil F, et al. [Assessing autonomic function in hypertrophic cardiomyopathy]. Arq Bras Cardiol 2005; 85: 388-396.

30. Bigger JT Jr, Fleiss JL, Steinman RC, Rolnitzky LM, Schneider WJ, Stein PK. RR variability in healthy, middle-aged persons compared with patients with chronic coronary heart disease or recent acute myocardial infarction. Circulation 1995; 91: 1936-1943.

31. Barbosa PR, Barbosa FJ, de Sa CA. [Effects of age, sex and 
coronary heart disease on the autonomic modulation of the heart]. Arq Bras Cardiol 1996; 67: 325-329.

32. Nunes NSV. [Contribution to the study of heart rate variability in children without evidences of structural heart disease]. [Master's thesis]: Cardiologia Clínica, Universidade Federal Fluminense, Rio de Janeiro, RJ, Brazil. Available at http:// www.bireme.br under request; 2002.

33. Atiga WL, Calkins H, Lawrence JH, Tomaselli GF, Smith JM, Berger RD. Beat-to-beat repolarization lability identifies patients at risk for sudden cardiac death. J Cardiovasc Electrophysiol 1998; 9: 899-908.

34. Saidi AS, Moodie DS, Garson A Jr, Lipshultz SE, Kaplan S, Lai WW, et al. Electrocardiography and 24-hour electrocardiographic ambulatory recording (Holter monitor) studies in children infected with human immunodeficiency virus type 1. The Pediatric Pulmonary and Cardiac Complications of Vertically Transmitted HIV-1 Infection Study Group. Pediatr Cardiol 2000; 21: 189-196.

35. Browne KF, Prystowsky E, Heger JJ, Chilson DA, Zipes DP. Prolongation of the Q-T interval in man during sleep. Am J
Cardiol 1983; 52: 55-59.

36. Yeragani VK, Berger R, Pohl R, Balon R. Effect of age on diurnal changes of 24-hour QT interval variability. Pediatr Cardiol 2005; 26: 39-44.

37. Wang $\mathrm{H}$, Lu Y, Wang Z. Function of cardiac M3 receptors. Auton Autacoid Pharmacol 2007; 27: 1-11.

38. Medei E, Pedrosa RC, Benchimol Barbosa PR, Costa PC, Hernandez CC, Chaves EA, et al. Human antibodies with muscarinic activity modulate ventricular repolarization: basis for electrical disturbance. Int J Cardiol 2007; 115: 373-380.

39. Montague TJ, Finley JP, Mukelabai K, Black SA, Rigby SM, Spencer CA, et al. Cardiac rhythm, rate and ventricular repolarization properties in infants at risk for sudden infant death syndrome: comparison with age- and sex-matched control infants. Am J Cardiol 1984; 54: 301-307.

40. Busti AJ, Tsikouris JP, Peeters MJ, Das SR, Canham RM, Abdullah SM, et al. A prospective evaluation of the effect of atazanavir on the QTc interval and QTc dispersion in HIVpositive patients. HIV Med 2006; 7: 317-322. 